\title{
COMPARING G-FORCE MEASUREMENT BETWEEN A SMARTPHONE APP AND AN IN-VEHICLE ACCELEROMETER
}

\author{
Johnathon P. Ehsani \\ Department of Health Policy and Management \\ Johns Hopkins Bloomberg School of Public Health \\ Baltimore, MD, USA \\ Email: Johnathon.ehsani@jhu.edu \\ Fearghal O'Brien \\ School of Business, National College of Ireland \\ Dublin, Ireland \\ Email: obrienfk@tcd.ie \\ Bruce Simons-Morton \\ Health Behavior Branch \\ Eunice Kennedy Shriver National Institute of Child Health and Human Development, \\ National Institutes of Health \\ Bethesda, MD, USA \\ Email: mortonb@exchange.nih.gov
}

\begin{abstract}
Summary: Due to their widespread adoption, smartphone applications (apps) could allow for a simple, low-cost assessment of driving behavior on a population scale. A number of existing apps are capable of measuring g-forces while driving, but few evaluations have been conducted to determine their accuracy. The goal of this study was to compare the measurement of g-forces between two devices: a custom-built smartphone app and an in-vehicle device that is currently used for research purposes (DAS). The test occurred under experimental conditions on a test track, where a vehicle, equipped with both the DAS and a smartphone with the app installed, performed a number of different acceleration events (e.g. hard-braking, sharp turning, etc.) under controlled conditions. We found that the app captured data that followed the same overall pattern of the DAS, but had a lower amplitude of measurement and a lower signal-to-noise ratio in the data. In general, the strength of the association between the app and DAS improved as the velocity of the events increased (though this was not true for all maneuvers). The correlations between the app and DAS were weaker for other maneuvers, and this may be due to delays in registering the maneuver. These findings indicate that a smartphone application did not register driving maneuvers in the same way that a dedicated in-vehicle device recorded them. Smartphones are ubiquitous and could represent a valuable driving research tool, however steps such as validation and testing are required, before they can be deployed in field trials.
\end{abstract}

\section{INTRODUCTION}

Motor vehicle crashes are the leading cause of death and a leading cause of injury for teenagers in the United States. In 2014, the most recent year data are available, approximately 2,500 teenagers in the United States aged 16-19 were killed, accounting for one third of all deaths in this age group[1]. A naturalistic driving study found that teenage drivers' elevated gravitation force (g-forces) event rates predicted their at-fault crashes [2]. Specifically, a composite measure of elevated g-force event rates (g-forces/miles driven) in the preceding month showed strong prediction of at-fault crashes and near-crashes in the following month. In that study, lateral and 
longitudinal acceleration and yaw, were measured by accelerometers installed in the vehicles of the research subjects.

Today, accelerometers have become ubiquitous due to their presence in smartphones. In 2013, three out of four teenagers in the U.S. owned a smartphone [3]. Smartphones are universally equipped with accelerometers, and GPS technology. Previous studies have demonstrated that smartphone accelerometers are a valid tool to measure other locomotion behaviors such as physical activity [4-6]. A number of existing smartphone applications (apps) are capable of measuring g-forces while driving. Automatic ${ }^{\mathrm{TM}}$ (2014) generates a driving score based on g-force events, and provides feedback to users to reduce gas consumption. TimetoDrive ${ }^{\mathrm{TM}}$ (2012) captures hard stops, and records the amount of driving.

Few evaluations have been conducted to determine the accuracy of these apps. Due to their proprietary nature, data that would allow for the assessment of g-force events and rates are not available to users or researchers. The use of widely available technology could extend the potential for research on risky driving to previously unmeasured populations. This would represent a new approach to understanding driving risk, using objective measures that are known to predict crashes. The purpose of this study was to compare measures of lateral and longitudinal acceleration from a custom-built smartphone app to a widely used in-vehicle device that measures acceleration.

\section{METHODS}

A smartphone app was developed for use on a Samsung Galaxy S5, running the Android 4.4 (KitKat) operating system [7]. The specifications for the app included the ability to measure the amount of driving (by trip duration and distance); lateral and longitudinal acceleration at multiple intensities; store these events in a database; and allow downloading of stored events to a database. The app was pilot tested and refined based in small-scale field tests in Washington D.C.

The ability of the app to measure lateral and longitudinal acceleration was compared with a widely used research tool for measuring g-force events; the Nextgen Data Acquisition System (DAS) developed at the Virginia Tech Transportation Institute (VTTI). The DAS uses the LSM303DLM 3-axis (3D) digital linear accelerometer, which also include a 3D digital magnetic sensor [8]. The device is factory calibrated for linear acceleration sensitivity, and linear acceleration level. According to the manufacturer, the adjustment values necessary for accurate measurement are stored inside the device in non-volatile memory. When the device is turned on, these parameters are downloaded into the registers to be used during normal operation, which allows the use of the device without further calibration.

The test occurred under experimental conditions on the Smart Road at Virginia tech [9]. The Smart Road is a 2.2-mile (3.5-kilometer), controlled-access closed-course system with a two-lane road constructed on a hillside. This private road segment allowed for a vehicle, equipped with both the DAS and a smartphone with the app installed, to undergo a number of different acceleration events (e.g. hard-braking, sharp turning, etc.) under controlled conditions, according to an experimental protocol. 


\section{Experimental protocol}

The app was installed on a smartphone that was mounted vertically (portrait position) on the windscreen inside the vehicle, alongside the DAS. A second phone (without the app installed) provided feedback of g-forces during driving maneuvers to the driver (Figure 1). The experimental drive was divided into four driving maneuvers: cornering left and right around a traffic circle, braking while driving in a straight line, accelerating from a stationary position, turning left and right at an intersection. Each maneuver was executed to reach a target level of force in order to determine the ability of the app to measure g-forces of different magnitudes (Table 1). The app and the DAS were re-started for each new task.

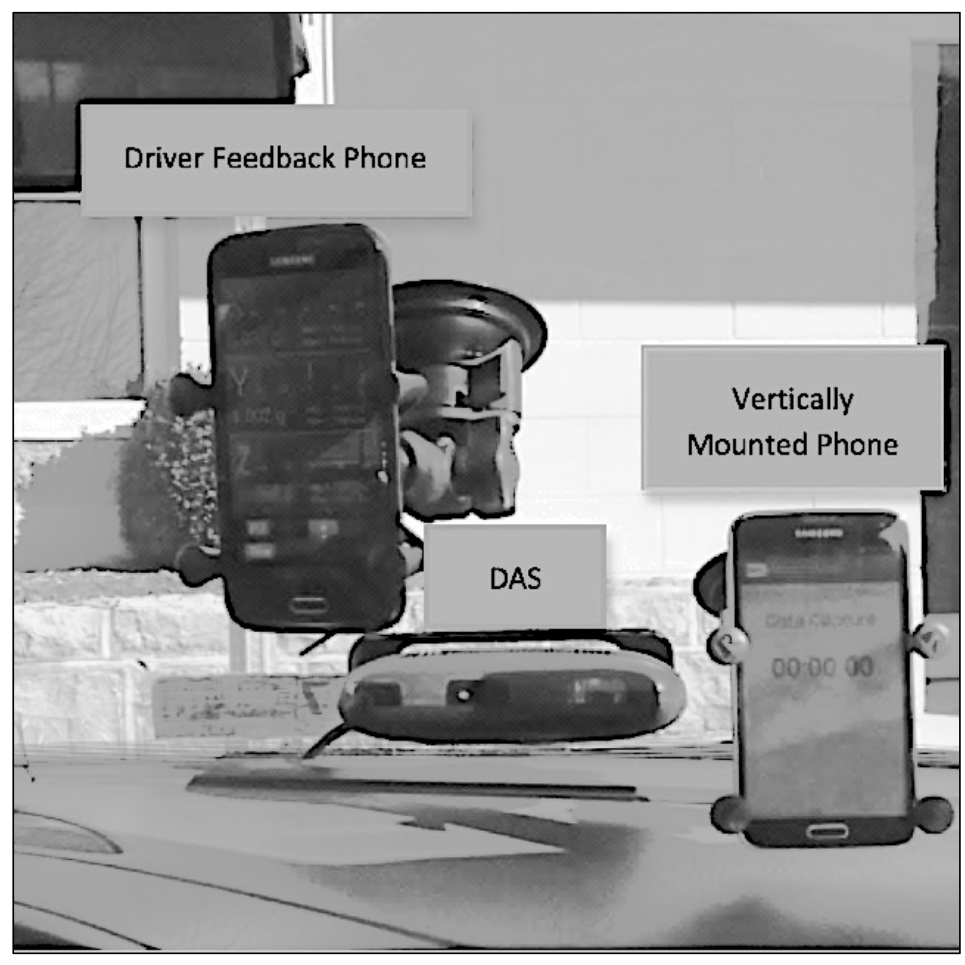

Table 1. Driving maneuvers and target G-forces

\begin{tabular}{|c|c|}
\hline Driving Maneuver & Target g-force \\
\hline \multicolumn{2}{|l|}{ Cornering } \\
\hline Mild Left & $0.2-0.3 \mathrm{G}$ 's \\
\hline Moderate Left & $0.3-0.4$ G's \\
\hline Hard Left & $0.5-0.6$ G's \\
\hline Mild Right & $0.2-0.3$ G's \\
\hline Moderate Right & $0.3-0.4$ G's \\
\hline Hard Right & $0.5-0.6$ G's \\
\hline \multicolumn{2}{|l|}{ Braking } \\
\hline Mild & $0.4-0.5$ G's \\
\hline Moderate & $0.5-0.6$ G's \\
\hline Hard & $0.6-0.7$ G's \\
\hline \multicolumn{2}{|c|}{ Acceleration from stationary position } \\
\hline Mild & 0.2 G's \\
\hline Hard & $0.3-0.4$ G's \\
\hline \multicolumn{2}{|l|}{ Turns } \\
\hline Mild Left & $0.2-0.3$ G's \\
\hline Moderate Left & $0.4-0.5$ G's \\
\hline Hard Left & $0.6-0.7$ G's \\
\hline Mild Right & $0.2-0.3 \mathrm{G}$ 's \\
\hline Moderate Right & $0.4-0.5$ G's \\
\hline Hard Right & $0.6-0.7$ G's \\
\hline
\end{tabular}

Figure 1. Smartphones and DAS mounted in the test vehicle

\section{Data processing and analyses}

The timestamps and the axes of the data collected by each device were aligned and plots were generated for each maneuver. The DAS data required a correction factor to account for the fact that the accelerometer did not consistently register zero at rest. This correction factor was achieved by subtracting the mean of the observed values for each maneuver from the data (demeaning) [10]. Demeaning was applied to data collected by both the DAS and app. Spearman rank correlations were conducted between the app and DAS for longitudinal and lateral acceleration. In addition, the percentage of time each device registered g-forces above $0.2 \mathrm{G}$ 's for each maneuver was calculated to compare data collection of elevated g-force events. 


\section{RESULTS}

The correlation between the lateral acceleration measured by the app and the DAS varied widely according to the nature and intensity of the driving maneuver. The highest correlation between the app and the DAS was observed for cornering hard right, $r=.65$ (Table 2 and Figure 2). For both left and right cornering, the strength of the correlation increased as the speed of the maneuver. However, the association was weaker for braking, acceleration and turning. In general, the patterns of acceleration recorded by the app and the DAS were similar, although the app consistently measured acceleration at a lower amplitude and with a lower signal-to-noise ratio (which is likely to be due to greater vibration). This is pattern is evident in Figure 2, which is the lateral acceleration plot from turning right at the highest speed (hard right). This plot is typical of the plots from several maneuvers, and reflected by the DAS recording a higher percentages of time where lateral acceleration was greater than $0.2 \mathrm{G}$ 's.

Table 2. Lateral Acceleration Correlation and Percent Time Above 0.2 G's for DAS and App

\begin{tabular}{|c|c|c|c|}
\hline \multirow[t]{2}{*}{ Driving Maneuver } & \multirow{2}{*}{$\begin{array}{c}\text { Correlation between } \\
\text { DAS and App* }\end{array}$} & \multicolumn{2}{|c|}{ Percent time $>0.2$ G's* } \\
\hline & & DAS & App \\
\hline \multicolumn{4}{|l|}{ Cornering } \\
\hline Mild Left & 0.42 & 0.06 & 0.00 \\
\hline Moderate Left & 0.49 & 0.18 & 0.00 \\
\hline Hard Left & 0.58 & 0.37 & 0.03 \\
\hline Mild Right & 0.52 & 0.08 & 0.00 \\
\hline Moderate Right & 0.59 & 0.24 & 0.00 \\
\hline Hard Right & 0.65 & 0.32 & 0.03 \\
\hline \multicolumn{4}{|l|}{ Braking } \\
\hline Mild & 0.10 & 0.00 & 0.01 \\
\hline Moderate & 0.14 & 0.00 & 0.00 \\
\hline Hard & 0.05 & 0.05 & 0.00 \\
\hline \multicolumn{4}{|c|}{ Acceleration from stationary position } \\
\hline Mild & 0.06 & 0.00 & 0.00 \\
\hline Hard & 0.04 & 0.00 & 0.03 \\
\hline \multicolumn{4}{|l|}{ Turns } \\
\hline Mild Left & 0.15 & 0.01 & 0.00 \\
\hline Moderate Left & 0.26 & 0.07 & 0.01 \\
\hline Hard Left & 0.17 & 0.07 & 0.03 \\
\hline Mild Right & 0.09 & 0.01 & 0.00 \\
\hline Moderate Right & 0.08 & 0.04 & 0.02 \\
\hline Hard Right & 0.02 & 0.04 & 0.03 \\
\hline
\end{tabular}


Figure 2. Lateral Acceleration Time Series for Turning Hard Right Recorded by App and DAS*

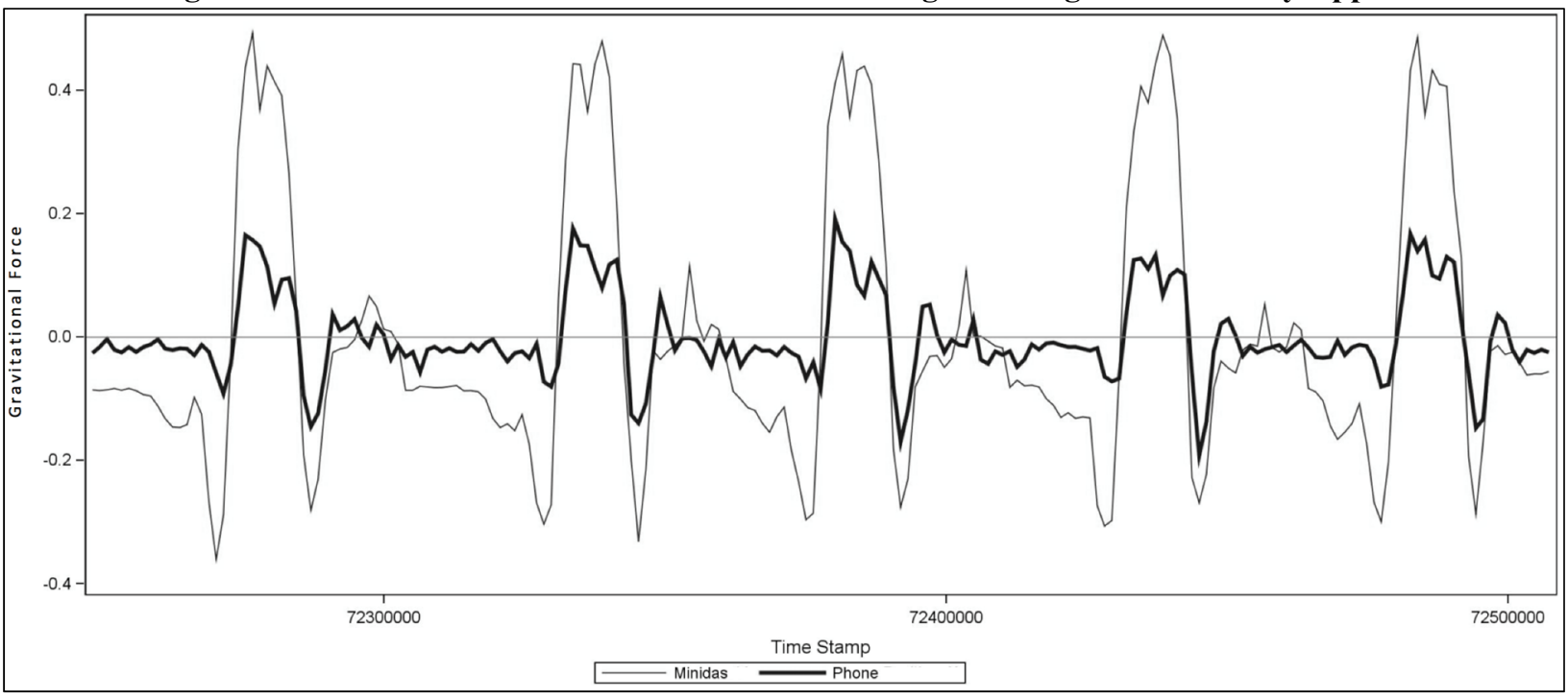

*Time series of lateral acceleration for turning hard right only. The correlation for this maneuver was $r=0.65$

The correlation between the app and DAS for longitudinal acceleration ranged from moderate to weak (Table 3). During mild and hard acceleration, the association between the app and the DAS was highest ( $r=.36$ and 44 respectively). The correlation was weaker for braking, although the observed differences appear to be due to a delay in the app recording the events, relative to the DAS (figure 3). The app and DAS had higher correlations during left cornering and left turns, compared to right cornering and turns. The DAS also registered a consistently higher percentage of time when the longitudinal acceleration was greater than $.2 \mathrm{G}$ 's.

Table 3. Longitudinal Acceleration Correlation and Percent Time Above 0.2 G's for DAS and App

\begin{tabular}{|c|c|c|c|}
\hline \multirow[t]{2}{*}{ Driving Maneuver } & \multirow{2}{*}{$\begin{array}{l}\text { Correlation between } \\
\text { DAS and App }\end{array}$} & \multicolumn{2}{|c|}{ Percent time above 0.2 G's } \\
\hline & & DAS & App \\
\hline \multicolumn{4}{|l|}{ Cornering } \\
\hline Mild Left & 0.29 & 0.03 & 0.00 \\
\hline Moderate Left & 0.31 & 0.05 & 0.00 \\
\hline Hard Left & 0.34 & 0.11 & 0.02 \\
\hline Mild Right & 0.05 & 0.06 & 0.00 \\
\hline Moderate Right & 0.08 & 0.06 & 0.01 \\
\hline Hard Right & 0.04 & 0.10 & 0.02 \\
\hline \multicolumn{4}{|l|}{ Braking } \\
\hline Mild & 0.21 & 0.21 & 0.09 \\
\hline Moderate & 0.27 & 0.40 & 0.11 \\
\hline Hard & 0.13 & 0.22 & 0.11 \\
\hline \multicolumn{4}{|c|}{ Acceleration from stationary position } \\
\hline Mild & 0.36 & 0.04 & 0.00 \\
\hline Hard & 0.44 & 0.28 & 0.05 \\
\hline \multicolumn{4}{|l|}{ Turns } \\
\hline Mild Left & 0.20 & 0.00 & 0.00 \\
\hline Moderate Left & 0.32 & 0.01 & 0.01 \\
\hline Hard Left & 0.33 & 0.07 & 0.02 \\
\hline Mild Right & 0.14 & 0.02 & 0.00 \\
\hline Moderate Right & 0.23 & 0.08 & 0.01 \\
\hline Hard Right & 0.23 & 0.11 & 0.01 \\
\hline
\end{tabular}


Figure 3. Longitudinal Acceleration Time Series for Hard Braking Recorded by App and DAS*

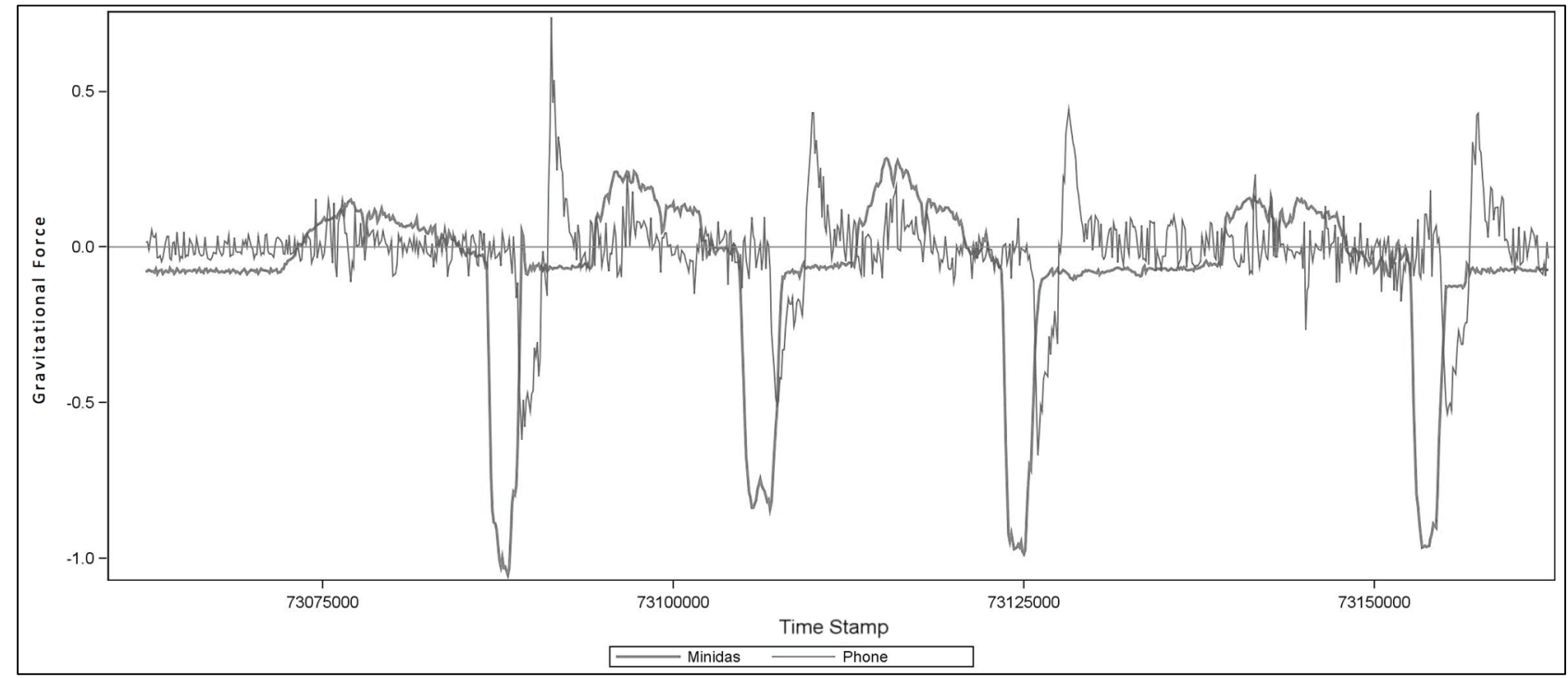

*Time series of longitudinal acceleration for hard braking only. The correlation for this maneuver was $r=0.13$

\section{DISCUSSION}

The goal of this study was to compare the measurement of g-forces between two devices: a custom-built smartphone app and an in-vehicle device that is currently used for research purposes. While neither device was validated against a known gold-standard, the purpose of the experiment was to determine the capabilities and limits of the app, for the purpose of identifying where future modifications and improvements could be made.

We found that the app captured data that followed the same overall pattern of the DAS, but had a lower amplitude of measurement and lower signal-to-noise ratio. The app had a high correlation with the DAS on certain driving maneuvers, such as left cornering and turning, and rapid acceleration from a stationary position. In general, the strength of the association between the app and DAS improved as the velocity of the events increased (though this was not true for all maneuvers). This is a promising finding, as the driving maneuvers of interest tend to be those at highly elevated g-forces. The correlation between the app and DAS were weaker for other maneuvers, and this may be due to a delay in registering the maneuver.

Provided they are able to deliver a reliable and accurate measurement of driving behavior, smartphones could be used by commercial fleets, insurance companies and parents of teenage drivers. These findings indicate several changes are necessary before a smartphone app can be used for research purposes. These include investigation of the hardware or software-related reasons for the lower amplitude data and event-detection delays, and signal filtering to eliminate the high frequency content in the data collected by the app.

\section{CONCLUSION}

Due to their widespread adoption, smartphone applications could allow for a simple, low-cost assessment of driving behavior on a population scale. We found that a custom-built app was able 
to measure certain driving maneuvers well, but had some limitations. With the necessary modifications, the app could be refined and used in a small-scale field study.

\section{ACKNOWLEDGEMENT}

This research was supported by the Intramural Research Program of the NICHD, contract \# N01HD-5-3405.

\section{REFERENCES}

Centers for Disease Control and Prevention. Data and Statistics (WISQARS): Cost of Injury Reports. 2016 [cited 2016 10th November]; Available from: http://www.cdc.gov/injury/wisqars/fatal.html.

Simons-Morton, B.G., et al., Do Elevated Gravitational-Force Events While Driving Predict Crashes and Near Crashes? American Journal of Epidemiology, 2012. 175(10): p. 1075-1079.

Neilsen. Ring the bells: More smartphones in students' hands ahead of back-to-school season. 2013 [cited 2014 2nd December]; Available from: http://www.nielsen.com/us/en/newswire/2013/ring-thebells-more-smartphones-in-students-hands-ahead-of-back.html.

Nolan, M., J.R. Mitchell, and P.K. Doyle-Baker, Validity of the Apple iPhone iPod Touch as an accelerometer-based physical activity monitor: a proof-of-concept study. Journal of physical activity \& health, 2014. 11(4): p. 759-769.

Khan, A.M., et al. Human Activity Recognition via an Accelerometer-Enabled-Smartphone Using Kernel Discriminant Analysis. in Future Information Technology (FutureTech), 2010 5th International Conference on. 2010.

Kirwan, M., et al., Design, Development, and Formative Evaluation of a Smartphone Application for Recording and Monitoring Physical Activity Levels: The 10,000 Steps: StepLog. Health Education \& Behavior, 2013. 40(2): p. 140-151.

Google, KitKat 4.4. 2014.

STMicroelectronics, LSM303DLM Sensor module: 3-axis accelerometer and 3-axis magnetometer. 2017, ST.com: Geneva, Switzerland.

Virginia Polytechnic Institute and State University. The Virginia Smart Road. 2015 [cited 2015; Available from: http://www.vtti.vt.edu/facilities/virginia-smart-road.html. 